\title{
Simulation codes for high brightness electron beam free-electron laser experiments
}

\author{
Luca Giannessi \\ Ente per le Nuove Tecnologie, l'Energia e l'Ambiente, UTS Tecnologie Fisiche Avanzate, \\ Via Enrico Fermi 45, 00044 Frascati, Rome, Italy \\ (Received 27 January 2003; published 10 November 2003)
}

\begin{abstract}
The high brightness electron beam required by a short wavelength self-amplified spontaneous emission free-electron laser (FEL) may be reached only with an accurate design of the beam dynamics from the generation in the $\mathrm{rf}$ injector up to the undulator. The beam dynamics is affected by strong selfconsistent effects at injection, in the compression stage, and during the FEL process. The support of numerical simulations is extensively used in the predictions of the beam behavior in these nonlinear dynamical conditions. I present a review of available simulation techniques, currently exploited in the design of short wavelength free-electron lasers.
\end{abstract}

DOI: 10.1103/PhysRevSTAB.6.114802

PACS numbers: 52.59.Sa, 41.60.Cr, 41.75.Lx

\section{INTRODUCTION}

We usually refer to a high quality $e$ beam as a beam with large brightness

$$
B_{n} \equiv \frac{2 I}{\varepsilon_{n x} \varepsilon_{n y}}
$$

i.e., high current and small normalized emittances. From a technical point of view, the quality of the electron beam is the main limiting factor in reaching short wavelengths with a self-amplified spontaneous emission (SASE) freeelectron laser (FEL). A proper understanding of the dynamics is essential in the design of short wavelength SASE FEL devices, where the constraints on the beam "quality" are very stringent. In this paper we review the most common techniques developed to study the beam behavior in strongly nonlinear conditions. In existing SASE FEL projects, low emittance beams are obtained within the present state of the art rf photocathode injectors and the peak current is increased by longitudinal compression. In these processes, as well as in the FEL amplification process itself, the nonlinear interaction of the beam with its self-fields plays a significant role.

The three dynamical regimes that will be analyzed are

(i) beam generation,

(ii) compression,

(iii) SASE FEL interaction.

In all these regimes we have to predict the behavior of the same physical system, consisting of an ensemble of charged particles which interacts with electromagnetic fields. Despite these common roots, there is no unique method for the solution of these problems and specialized techniques have been developed. The main difficulty is a common one and consists of the large number of electrons contained in a single bunch of charge. The typical $e$-beam charge is of the order of $10^{-9} \mathrm{C}$, corresponding to $\sim 10^{10}$ electrons. A multiparticle system of this size cannot be efficiently simulated in all its complexity, even in a state of the art, parallel computer. The widely used solution is that of simulating a reduced number of particles, with a scaled charge, each representing a large number of real electrons. The introduction of these macroparticles has some unpleasant consequences that need to be properly treated in a correct numerical implementation. This fact can be shown with a simple example. Let us consider a Gaussian bunch with rms $\sigma_{\text {beam }}$, represented by a number $N$ of macroparticles. The charge density, defined as

$$
\rho(x, N)=\frac{1}{N} \sum_{j=1}^{N} \delta\left(x-x_{j}\right)
$$

in Fourier space becomes

$$
\rho(x, N)=\frac{1}{N} \sum_{j=1}^{N} e^{i k x_{j}}
$$

that, in the limit of $N \rightarrow \infty$, reduces to the Fourier transform of the Gaussian distribution

$$
\rho(x)=\exp \left(-\frac{k^{2} \sigma_{\text {beam }}^{2}}{2}\right)
$$

In Fig. 1, the spectrum of the distribution obtained from Eq. (3), for $N=100$ and $N=10^{4}$, is shown. At low wave vector $k$, the behavior is that of a smooth distribution, correctly reproducing the Gaussian. At high $k$, the phase factors in the exponent of Eq. (3) are uncorrelated and the amplitudes of the corresponding Fourier components scale as $1 / \sqrt{N}$. The effect of a reduced number of macroparticles in the representation of a charge distribution is evident. In a real beam the amplitude of this high frequency "noise," commonly referred to as "shot noise," is orders of magnitude lower than in the case of a "simulated" beam. A "noisy" charge distribution by itself should not be considered as a problem. It becomes a problem when the electromagnetic fields that are driving the dynamics of the whole system depend on the properties of such distribution. This feedback loop, charge distribution $\rightarrow$ fields $\rightarrow$ charge distribution, must be 


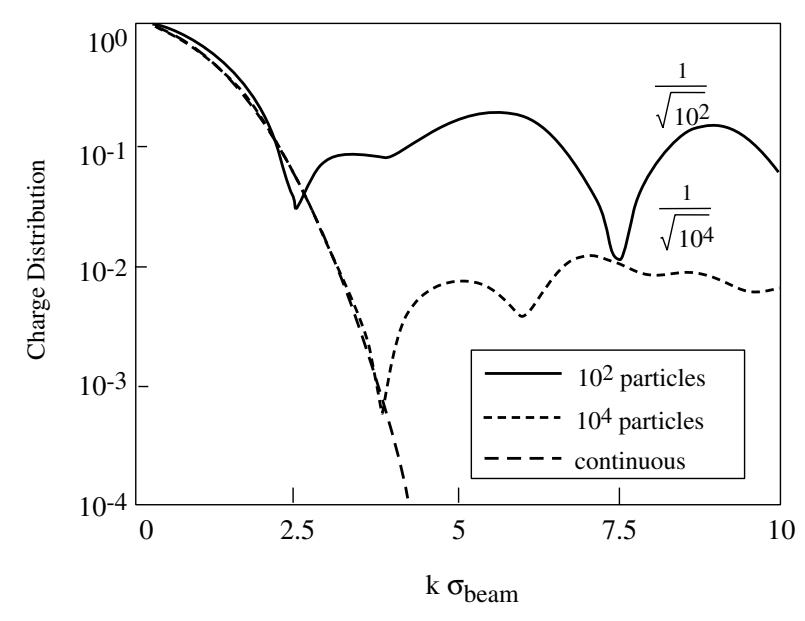

FIG. 1. Spectrum of charge distribution for an analytical continuous distribution and for a distribution sampled with $10^{2}$ and $10^{4}$ macroparticles. The charge distribution is normalized to unity.

simulated only for those frequency components where the distribution has the correct spectral behavior of the physical distribution. The other frequencies must be suppressed, otherwise the simulation will produce unphysical results.

This frequency selection is one of the main distinguishing features of the three regimes of operation mentioned above. In an injector we have bunch lengths of a few picoseconds, interacting at $\mathrm{mm} / \mathrm{sub} \mathrm{mm}$ wavelengths. In a compressor the bunch length is reduced to a few tens of microns, and the coupling wavelength can scale down to the micrometer range. These are broad-band coupled systems where the high frequency components should be filtered for a correct numerical representation. In these broad-band conditions, filtering is generally obtained by giving a finite size to the macroparticles [1-3]. The Fourier transform of a "Gaussian" particle of rms extension in space $\sigma_{\text {part }}$ is proportional to $\exp \left(-k^{2} \sigma_{\text {part }}^{2} / 2\right)$. The effect of a spatial extension is that of a low band filter. A similar result may be accomplished for radiation fields in the coherent synchrotron radiation (CSR) case by spreading the particles in momentum space [4]. In a free-electron laser, the coupling fields may have very high frequency components, but in a very narrow frequency bandwidth, which is proportional to the Pierce parameter [5]. The input phase space is prepared to reduce the harmonic content in this spectral window with a "quiet start" procedure (see Sec. IVA) and the narrowness of the spectrum is exploited in simplifying the equations for the fields and the particle dynamics.

The frequency responses typical of the various regimes are exploited in the numerical implementations to obtain correct results with a reduced number of macroparticles. While all the most widely known numerical models are based on macroparticles adopting different techniques to reduce the associated numerical noise, not all the meth- ods have corresponding variables for the description of the fields. We can indeed distinguish between:

Differential methods.-The fields are independent variables of the problem. These methods require the simultaneous integration of the Maxwell's equations and the Lorentz force equation. Particles in cells (PIC) codes belong to this family. The fields are known on a mesh filling the simulation space-time with some boundary conditions. Field sources are macroparticles which are assigned to the cells of the mesh according to some assignment procedure designed to limit the spectral content of pointlike macroparticles. Most of the codes devoted to FELs simulation are based on differential methods. The limited bandwidth of the resonant process allows in this case to cast the Maxwell's equations in terms of slowly varying components propagating with the $e$ beam (slowly varying envelope approximation).

Integral methods.-The electromagnetic fields are not independent variables of the problem. The interaction between particles is calculated according to "forces" derived from particle positions and velocities known at earlier times. Effects of plane walls can be included with the image charge method while radiative and retarded effects may still be included by the Lienard-Wiechert retarded potential formalism [6].

The first class of solvers allows the implementation of rigorous physical models, including "in principle" almost all the aspects of the problem, such as the interaction with walls of any shape, any kind of retarded effects associated with the finite "time of flight" of signals, radiative effects, etc. There is a price to be paid for this physical accuracy, i.e., Maxwell's equations are partial differential equations and the solver must follow some important prescriptions. The fields are indeed known on a mesh, characterized by a given spacing $\left(\delta_{x}, \delta_{y}, \delta_{z}\right)$. According to the Nyquist theorem there is a limit in the highest wave vector that can be represented by the mesh,

$$
k_{x, y, z}=\frac{1}{2 \delta_{x, y, z}}
$$

and consequently, in the highest frequency that can be supported by the mesh,

$$
\omega_{\max }=c \max \left(k_{x}, k_{y}, k_{z}\right)=\frac{c}{2 \min \left(\delta_{x}, \delta_{y}, \delta_{z}\right)} .
$$

Higher frequencies will appear as low frequency components. For the same reasons the time step size is limited by this maximum frequency by the relation

$$
\delta t<\frac{1}{\omega_{\max }} .
$$

There are other limitations to the time step length that are dependent on the order of the integration method, on the integration scheme, that may be implicit or explicit, on the dimensions of the problem (see Ref. [7], and references therein for further details), but the basic 
TABLE I. Typical mesh sizes for the simulation of the beam dynamics at the injection, compression, and FEL stages with PIC techniques.

\begin{tabular}{lccc}
\hline \hline & Injector & Compressor & FEL \\
\hline Simulation volume & $300 \mathrm{~cm}^{3}$ & $100 \mathrm{~cm}^{3}$ & $1 \mathrm{~mm}^{2} \times 1 \mu \mathrm{m}$ \\
Cutoff wavelength & $100 \mu \mathrm{m}$ & $10 \mu \mathrm{m}$ & $1 \AA$ \\
No. of Mesh vertices (3D) & $10^{7}$ & $10^{11}$ & $10^{16}$ \\
No. of Mesh vertices (2D) & $10^{5}$ & $10^{7}$ & $10^{10}$ \\
Integration time & $16 \mathrm{ps}$ & $60 \mathrm{ps}$ & $330 \mathrm{ps}$ \\
Maximum step length & $0.3 \mathrm{ps}$ & $0.03 \mathrm{ps}$ & $3 \times 10^{-19} \mathrm{~s}$ \\
No. of time steps & $3 \times 10^{3}$ & $10^{6}$ & $10^{12}$ \\
\hline \hline
\end{tabular}

condition (7) must be satisfied, longer time steps would cause lack of accuracy and in some cases instability of the solution. In Table I we have summarized typical numbers for the mesh and time step requirements in the three regimes of beam dynamics.

Assuming a limit of $10^{6}$ mesh vertices for a numerical implementation, only the simulation of a 2D injector seems practicable. In the example of Table I we have assumed typical numbers for a "short" wavelength SASE FEL. The mesh size can be reduced in this case by taking advantage of the narrow bandwidth of the FEL gain, with the slow wave approximation used by most FEL devoted codes. There have been however successful attempts in the simulation of FELs with PIC codes developed for plasma dynamics, in the long wavelength regime, and in conditions of reduced dimensions [8].

This paper is organized as follows. In the next section we will review the techniques used in the simulation of photoinjectors. According to the above discussion these techniques have been classified in terms of differential method and integral method based (Secs. II A and II B). This distinction is not appropriate in the description of the compressor stage considered in Sec. III. As shown in Table I the simulation of magnetic compression with a PIC code even in a 2D Cartesian mesh would require an extremely large number of mesh vertices. Furthermore the solution of the dynamics of highly relativistic charges coupled by Maxwell's equations in Cartesian geometry is seriously affected by the "decoherence" problem described in Sec. II A. For this reason the codes devoted to the simulation of compressors are based on integral methods. The formulation of the free-electron laser dynamics, on the other side, relays on the slow wave approximation and on the paraxial wave approximation, and almost all the techniques implemented in the simulation of free-electron lasers, which will be analyzed in Sec. IV, are based on differential methods, where the Maxwell's equations and Lorentz force equation have been recast in terms of slowly varying functions.

\section{CODES FOR INJECTOR DESIGN}

We consider an "injector" as all the elements required to bring the beam from rest energy in the lab frame up to an energy that is large enough to neglect effects of selfinteraction in a uniform motion condition. A typical layout of a "split" system is shown in Fig. 2.

An injector is characterized by

(i) a very high gradient, i.e., the beam becomes relativistic in the first half cell of the rf gun.

(ii) The electron pulse length is much shorter than the radio frequency period.

(iii) An rf injector is a "quasi"-axially symmetric device. Deviations from the axial symmetry may be due to an inhomogeneity of the cathode quantum efficiency, to dipolar terms in the accelerating fields, to an asymmetry of the laser spot, and to misalignments of the structure. Unless the study of one of these aspects is required, the assumption of axial symmetry greatly simplifies the problem by reducing the required number of macroparticles and (or) mesh points.

The dynamics of such a system has been extensively described in [9]. The request of minimizing the induced emittance growth is accomplished by tuning the frequency of the first plasma oscillation that the beam executes according to the internal space charge fields and to the focusing due to rf forces and to the solenoid. When this frequency is correctly matched, the emittance has a minimum at an energy high enough that the contribution of the betatron motion associated with the thermal emittance overcomes that of the laminar motion. In ideal conditions, i.e., a beam which is flat transversally and longitudinally, this "emittance compensation" procedure brings the emittance at the end, almost to the same

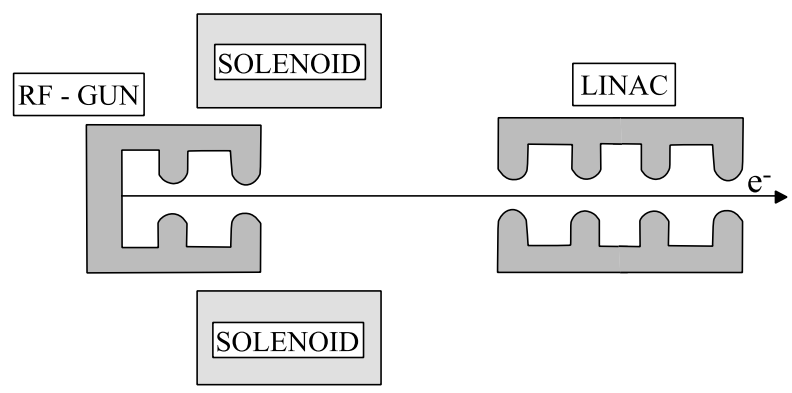

FIG. 2. Typical layout of an "injector." 
order of the initial thermal emittance. This emittance compensation concept originally introduced in [10] produced serious improvements in the beam brightness obtained at the injector exit [11] and, from the simulation point of view, substantially raised the requests in terms of numerical accuracy and resolution.

\section{A. Differential methods}

Even though some details of the beam dynamics in an rf injector may be affected by three-dimensional effects, an injector is substantially an axisymmetric device. Table I shows that the mesh size and time step length relevant to a PIC simulation of the beam dynamics in cylindrical symmetry make such a numerical implementation feasible. The restriction to cylindrical symmetry provides an even larger extra advantage in the formulation of the problem that goes beyond the simple reduction of particles/mesh points. In axial symmetry a closed subset of Maxwell equations, driven by the source $[\rho, J]$, can be fully described in terms of a scalar pseudopotential

$$
\Phi \equiv r \cdot H_{\varphi}(r, z, t)
$$

obeying the scalar wave equation

$$
\left[\frac{\partial^{2}}{\partial z^{2}}+\frac{\partial^{2}}{\partial r^{2}}-\frac{1}{\partial r}\right] \Phi-\frac{1}{c^{2}} \frac{\partial^{2} \Phi}{\partial t^{2}}=r \frac{\partial J_{z}}{\partial r}-r \frac{\partial J_{r}}{\partial z}
$$

Electric and magnetic fields are recovered from the function $\Phi$ according to

$$
\frac{d E_{r}}{c d t}=-\frac{1}{r} \frac{\partial \Phi}{\partial z}-J_{r}, \quad \frac{d E_{z}}{c d t}=-\frac{1}{r} \frac{\partial \Phi}{\partial r}-J_{z} .
$$

The advantage in this formulation goes beyond the fact that the solution can be obtained solving a scalar wave equation in place of vector equations. The main advantage is that this formalism allows one to get around the decoherence problems associated with Maxwell's equations in a conventional leapfrog integration scheme. In Cartesian geometry, the implementation of the method on a mesh with vertices of coordinates $\left(r_{k}, z_{l}\right)$ with $k=1 \cdots K, l=$ $1 \cdots L$, assumes the form

$$
\begin{aligned}
\vec{B}_{k, l}(t+\delta t)= & \vec{B}_{k, l}(t-\delta t)+\vec{g}\left[\vec{E}_{k \pm 1 / 2, l \pm 1 / 2}(t)\right], \\
\vec{E}_{k+1 / 2, l+1 / 2}(t+\delta t / 2)= & \vec{E}_{k+1 / 2, l+1 / 2}(t-\delta t / 2) \\
& +\vec{f}\left[\vec{B}_{k \pm 1, l \pm 1}(t), \vec{J}_{k \pm 1, l \pm 1}(t)\right],
\end{aligned}
$$

where we used the notation $U_{k, l}=U\left(r_{k}, z_{l}\right), U=E, B$, and where the functions $\vec{f}$ and $\vec{g}$ are the linear summation of the $\nabla \times \vec{B}+4 \pi \vec{J}$ and $\nabla \times \vec{E}$ terms, respectively. It is evident from Eq. (11) that magnetic and electric components of the fields are not known at the same time and in the same position. The error induced by this displacement is critical because of the electric and magnetic field cancellations in the transverse force, whose combined contributions scale with the particle's energy, as $1 / \gamma^{2}$. A lack of cancellation brings longitudinally correlated forces inducing a nonphysical emittance growth [12]. For this reason 3D particle in cell codes developed for plasma physics applications are not reliable in the simulation of high brightness relativistic beams. Alternative 2D cylindrical codes such as ITACA [13] and SPIFFE [14] have been developed and extensively used. The main limitations in their application are still related to the size of the mesh required in the simulation of short bunches. The mesh size is indeed grown with the introduction of the emittance compensation concept, both because of the larger spatial extension where the space charge dynamics must be followed and because of the increased numerical resolution required by the brightness improvement. As a final remark, we note that the frequency tuning and the field balancing between cavities in a multicell gun are all an integral part of the simulation and are affected by the beam parameters. The problems related to the determination of "external fields" and "space charge" fields are not fully separated as they are with integral methods, and from the practical point of view, the setup of a simulation may be in this case somewhat less handy.

\section{B. Integral methods}

The analysis of the beam dynamics developed in Ref. [9] has pointed out that a beam with a typical current of 100 A may still be in the space charge dominated condition even at quite high energies, exceeding $100 \mathrm{MeV}$. The request of such a high energy has extended longitudinally the "simulation volume" to several meters. At the same time the electron pulses of a few picoseconds produced with photocathodes have increased the harmonic content of space charge fields in the bunch, reducing the required step size of the mesh for a PIC code. Simulation of a long system with a small mesh period tends to become time consuming and less practical. According to the example shown in Table I these considerations are even more important in an extension to the 3D domain. Codes based on integral methods do not suffer from this limitation. The evaluation of the fields from the phase space coordinates of the particles is done only in the positions instantaneously occupied by the particles. Some codes, as TREDI [15] or ATRAP [16], take into account the effects due to the finite propagation velocity of signals by calculating the fields according to the Lienard-Wiechert formalism,

$$
\begin{aligned}
& \vec{E}=\frac{\vec{n}\left[(\vec{n}-\vec{\beta}) \frac{d}{d t} \vec{\beta}\right]}{(1-\vec{\beta} \cdot \vec{n})^{3}|\vec{R}|}+\left.\frac{(\vec{n}-\vec{\beta})\left[1-|\vec{\beta}|^{2}\right]}{(1-\vec{\beta} \cdot \vec{n})^{3}|R|^{2}}\right|_{\text {ret }}, \\
& \vec{B}=\left.\vec{n} \vec{E}\right|_{\text {ret }},
\end{aligned}
$$

evaluated at the retarded time

$$
t^{\prime}=t-\frac{\left|\vec{R}\left(t^{\prime}\right)\right|}{c} .
$$


This is accomplished in TREDI by storing in memory the histories of the macroparticle trajectories and by tracking the source coordinates back in time until the retarded condition (13) is satisfied. The effects of boundaries can be included only for flat walls by the image charge method. In practice only the cathode wall is considered. The fields used for the calculation of the dynamics are the superposition of given external fields and the self-fields from all the particles evaluated on a point to point basis. This field evaluation procedure relies on the choice of a "screening radius," the size of the macroparticles which limits the high frequency components. From the example of Fig. 1, we have a significant hint of the choice of this screening radius. The ideal wave vector cutoff is the one that leaves unchanged the global beam distribution at low $k$ and that efficiently cuts the noise in the high $k$ region. In this simple example based on a smooth Gaussian charge distribution, the transition frequency is located at $k_{\text {cut }}=$ $\left(1 / \sigma_{\text {beam }}\right) \sqrt{\ln (N)}$ where $N$ is the number of macroparticles. A particle extended in space with $\mathrm{rms} \sigma_{\text {part }}=$ $1 / k_{\text {cut }}$ shows the desired filtering properties. The situation is practically complicated by the fact that the beam distribution is not in general a Gaussian. Optimized emittance compensation is obtained with a charge distribution which is a homogeneous cylinder oriented along the $z$ axis. In this case the desired cutoff wave vector scales with the number of macroparticles as $k_{\mathrm{cut}} \propto \sqrt{N^{3}}$ but the shape of the charge distribution may also change in time with the beam evolution, and the correct choice of the screening radius remains somewhat arbitrary. The above scheme still shows some other drawbacks. The backward tracking procedure is time consuming, and in a point to point interaction scheme the number of evaluations scales as the square of the number of macroparticles. An improvement of the method consists of evaluating the fields on a mesh surrounding the beam and by interpolating these fields to the particle's positions. This allows one to simulate a larger number of macroparticles with a defined mesh size, but still the major issue of the approach is constituted by the computation time. A simplification is obtained in the "static" approximation used, e.g., in PARMELA [17], in GPT [18,19], in ASTRA [20], and in a slightly different flavor, in TREDI also (run in static mode). This approximation consists of the assumption that the beam relative energy spread is small, and that a reference frame where the beam may be considered at rest exists. The effect is that of neglecting the finite velocity of signal propagation within the bunch. The relative energy spread $\Delta \gamma / \gamma$ scales with the bunch length $\Delta \phi$, as [21]

$$
\frac{\Delta \gamma}{\gamma} \approx \frac{\alpha \Delta \phi}{1+\pi \alpha z / \lambda_{\mathrm{rf}}}<1
$$

where $\alpha=\left(\lambda_{\mathrm{rf}} / 2 \pi\right)(d \gamma / d z) \approx 1$ is proportional to the average accelerating gradient.
In these conditions (as, e.g., in PARMELA "SCHEFF" and "SPCH3D" modes), the self-fields are calculated by solving the Poisson equation for the electrostatic field in this moving frame. The fields are then transformed back to the laboratory frame where kicks to the particles are applied. In a photoinjector the electron bunch is short with respect to the rf period and when the beam becomes relativistic the approximation (14) becomes fully satisfied. The largest energy spread occurs in the proximity of the cathode, where the beam is not yet relativistic and the quasistatic approximation still works. The quasistatic approximation may be critical in the simulation of long bunches as in the case of rf thermoionic guns, where the longitudinal phase spread leads to a large energy spread at the cathode. In ASTRA the quasistatic assumption may be partially relaxed by considering macroparticles not completely at rest in the beam reference frame, but in nonrelativistic motion. With this approximation also magnetic fields are generated in the rest frame in addition to the electrostatic fields which are then transformed into the lab frame. This option can be switched off. A comparison of simulations obtained with this option on and off has not shown significant differences [22].

A very efficient algorithm is obtained in HOMDYN $[23,24]$, by considering a multienvelope model based on the time dependent evolution of a uniform bunch [25],

$$
\begin{aligned}
\frac{d^{2}}{d z^{2}} \sigma\left(z, \xi_{i}\right)+\frac{p^{\prime}\left(z, \xi_{i}\right)}{p\left(z, \xi_{i}\right)} \sigma\left(z, \xi_{i}\right)+K \sigma\left(z, \xi_{i}\right) \\
\quad=\frac{I\left(z, \xi_{i}\right) g\left(z, \xi_{i}\right)}{2 I_{0} p\left(z, \xi_{i}\right)^{3} \sigma\left(z, \xi_{i}\right)}+\frac{\varepsilon_{n, t h}^{3}}{p\left(z, \xi_{i}\right)^{2} \sigma\left(z, \xi_{i}\right)^{3}}, \\
i=1 \cdots N,
\end{aligned}
$$

where $I_{0}=\left(e c / r_{0}\right)$ is the Alfvén current, and where the electron bunch is sliced along the direction of propagation, as shown in Fig. 3. The coordinate $z$ in Eq. (15) represents the evolution along the beam line, and the coordinate $\zeta_{i} \equiv z_{i}-\beta_{i} c t_{i}$ indicates the position of a slice of the beam along the bunch. We have indicated with $I\left(z, \xi_{i}\right)$ the slice current, with $p\left(z, \xi_{i}\right)$ the slice longitudinal momentum and with $p^{\prime}\left(z, \xi_{i}\right)=(d / d z) p\left(z, \xi_{i}\right)$ its derivative in $z$. The function

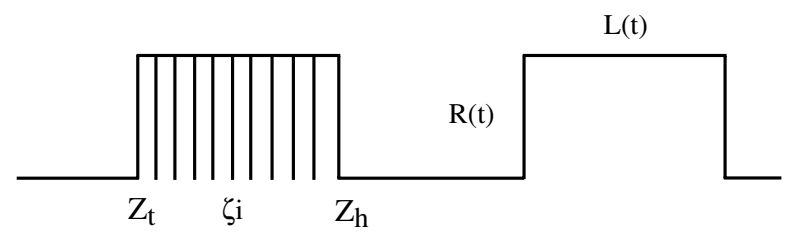

FIG. 3. Electron beam longitudinal profile and slicing procedure adopted in HOMDYN. The bunch shape is assumed cylindrical with length $L$ and radius $R$. The longitudinal coordinate of the $i$ th slice is indicated with $\zeta_{i}$ spanning from the bunch tail $Z_{t}$ to the bunch head $Z_{h}$. 


$$
\begin{aligned}
g\left(z, \zeta_{i}\right)= & \frac{1-\zeta_{i} / L(z)}{\sqrt{\left[1-\zeta_{i} / L(z)\right]^{2}+A\left(\bar{z}, \zeta_{i}\right)^{2}}} \\
& +\frac{\zeta_{i} / L(z)}{\sqrt{\left[\zeta_{i} / L(z)\right]^{2}+A\left(z, \zeta_{i}\right)^{2}}}, \\
A\left(z, \zeta_{i}\right) \equiv & \frac{R\left(z, \zeta_{i}\right)}{\gamma L(z)}
\end{aligned}
$$

represents the radial space charge interaction of a single slice with the whole beam, depending on the instantaneous bunch length and aspect ratio $A$. The above expression has been derived for a cylindrical beam of homogeneous density. The approximation in HOMDYN consists of considering this shape of the beam distribution unchanged along the beam line, except for the aspect ratio and length. In the ideal case the beam is indeed transversally uniform at the extraction, to preserve the linearity of the transverse fields. This uniformity is not preserved by the dynamics, but in the first stages of the acceleration, where the space charge forces dominate the modifications in the bunch shape are sufficiently small to allow HOMDYN to correctly reproduce the dynamics of the emittance compensation process. The beam emittances are calculated at each step as the projected emittances of all the slices of the beam,

$\Delta \varepsilon_{n}^{\mathrm{cor}}=\frac{1}{N} \sqrt{\sum_{i=l}^{N} \sigma\left(z, \zeta_{i}\right)^{2} \sum_{i=l}^{N} \sigma^{\prime}\left(z, \zeta_{i}\right)^{2}-\left[\sum_{i=i}^{N} \sigma\left(z, \zeta_{i}\right) \sigma^{\prime}\left(z, \zeta_{i}\right)\right]^{2}}$.

Despite the strong assumptions, HOMDYN allows a sufficiently accurate determination of the working point which usually depends on a large number of input parameters as input phase, solenoid field, solenoid position, etc. Furthermore the simulation results show only a weak dependence on the number of slices and a run with 40 slices of the first $10 \mathrm{~m}$ of the SLAC injector [25] lasts less than 1 min on a $2 \mathrm{GHz}$ Intel P-IV [26].

\section{Comparison between codes}

In Fig. 4 we have shown a comparison of simulations of a split configuration similar to the one of Fig. 2, obtained with ITACA, PARMELA, and HOMDYN. The dashed lines represent the radial emittance and the continuous lines represent the beam envelope. With the assumption of unchanged beam shape, HOMDYN preserves the linearity of transverse fields for each slice along the integration path. For this reason it reaches the lowest emittance. The behavior of the emittance relevant to ITACA was partially affected by the size of the mesh. The trend observed has shown an improvement of the agreement with a finer mesh [27]. The differences in waist positions and emittances minima suggest also that a slight difference in the definition of the solenoid field may have played a role (there is a strong sensitivity to the magnetic field first integral). In Fig. 5 it is shown a comparison between
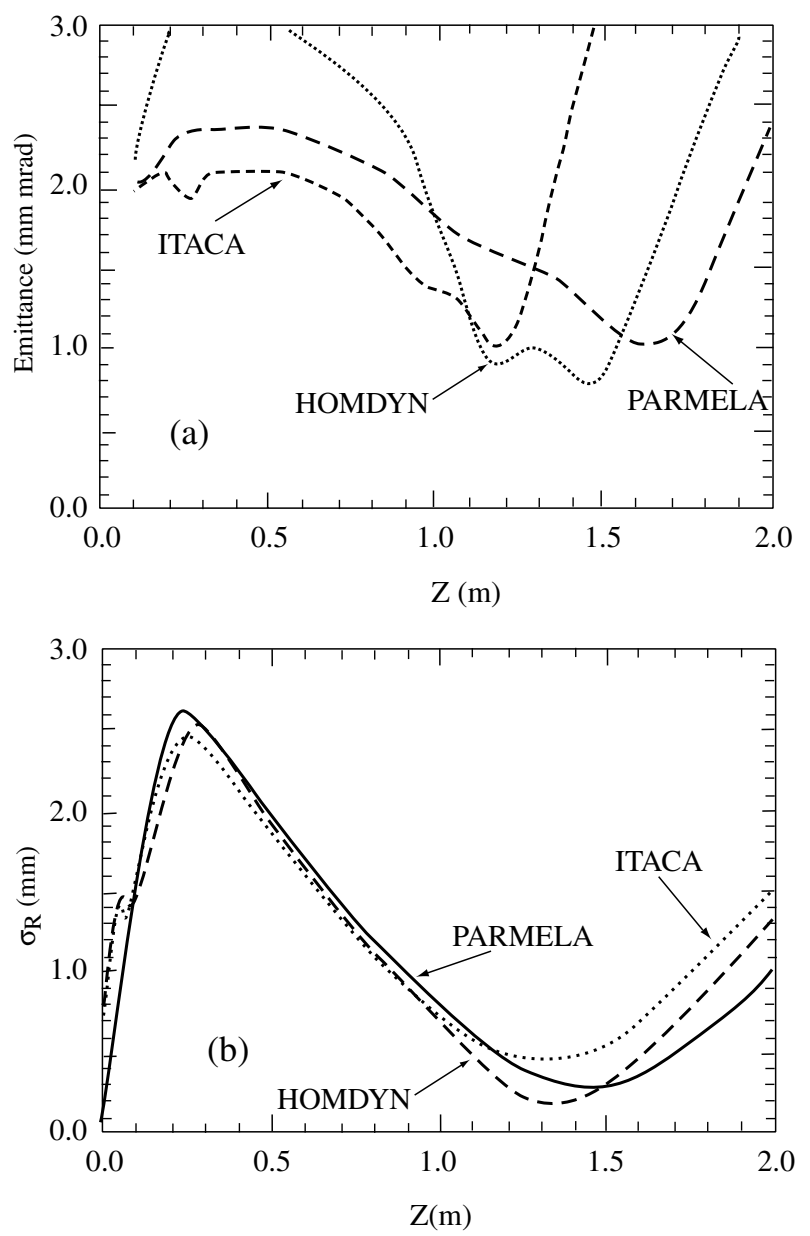

FIG. 4. Comparison between ITACA, PARMELA, and HOMDYN obtained in a standard split injector configuration as the one shown in Fig. 2. The behaviors of the normalized emittance and of the beam rms envelope versus the longitudinal coordinate are shown in (a) and (b), respectively.

PARMELA and HOMDYN for a split system including two traveling wave (TW) linac sections, with the parameters of Table II.

The approximation of a uniform bunch preserved along the beam line on which HOMDYN rely produces a more pronounced effect of emittance compensation. The agreement is however reasonable and the position of the minimum of the emittance before the linac is approximately reproduced. A comparison of HOMDYN with ASTRA has shown a similar behavior [28]. In Fig. 6 a comparison between PARMELA and TREDI with the same parameters is shown for the first $1.7 \mathrm{~m}$. Despite the differences in the models, the minimum projected emittance differs by only $10 \%$. The working point of Table II which has been optimized with PARMELA and HOMDYN appears as not perfectly optimized in the TREDI simulation. Differences are probably due to the different algorithmic representation of the fields (mapping and interpolation) and, in part, to the retarded effects which are included in TREDI. More details may be found in Ref. [29]. 

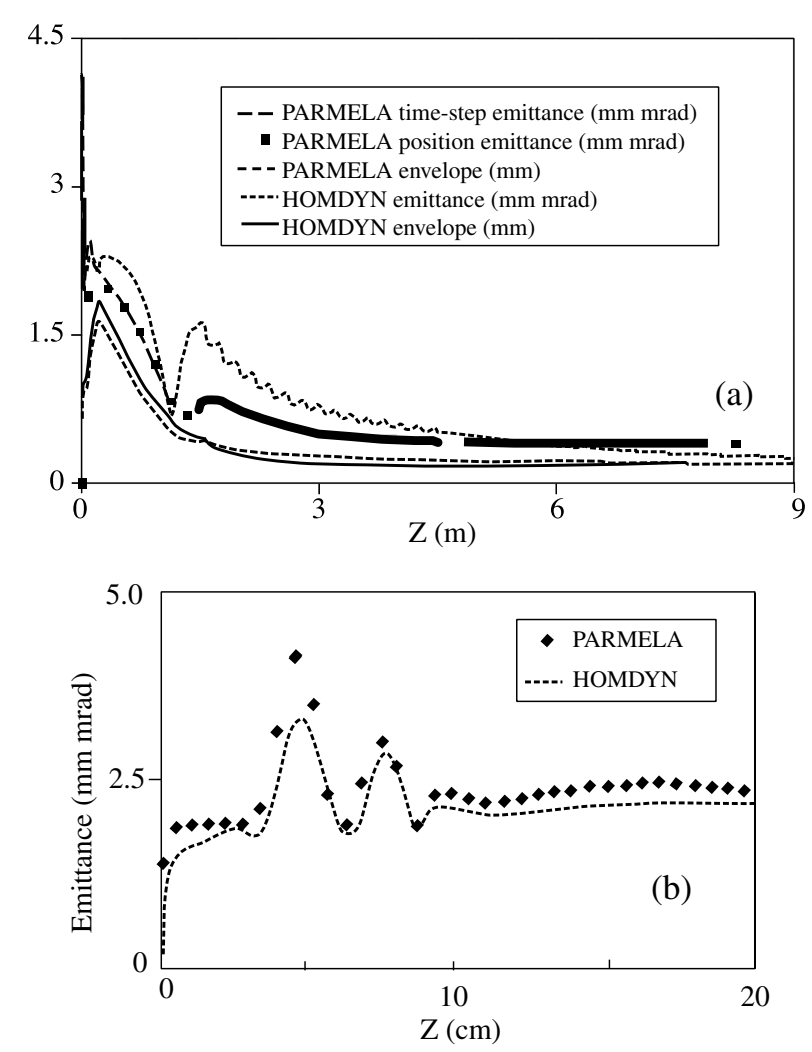

FIG. 5. Comparison between PARMELA and HOMDYN with the parameters listed in Table II. The simulation with PARMELA has been obtained with $10^{4}$ macroparticles, a time step of $0.1^{\circ}$, and a mesh of $20(r) \times 400(z)$ points (SCHEFF mode). The upper plot represents the first $20 \mathrm{~cm}$ of the simulation.

One of the targets of the analysis of Ref. [30], where PARMELA (LANL and UCLA) has been compared to other PIC codes, was the study of the limits of the quasistatic approximation. The results have shown an overestimation of the PARMELA versus PIC codes as MAGIC2D [31] emittances, by about $20 \%$. The conclusions attributed part of this difference to the quasistatic approximation adopted in PARMELA. Another comprehensive comparison between several codes including HOMDYN, ASTRA, PARMELA, and TREDI has been presented in Ref. [32] consisting of the simulation of an $s$-band $\mathrm{rf}$ gun with an emittance compensation solenoid, $1 \mathrm{nC}$ of

TABLE II. Set of parameters for the simulation shown in Fig. 5.

\begin{tabular}{lc}
\hline \hline Gun & BNL, 1.6 cells \\
Charge & $1 \mathrm{nC}$ \\
Spot radius & $1 \mathrm{~mm}$ \\
Laser pulse (flat) & $10 \mathrm{ps}$ \\
Peak field & $140 \mathrm{MeV} / \mathrm{m}$ \\
Linac & $2 \mathrm{TW} \mathrm{sections}$ \\
Linac gradient & $25 \mathrm{MeV} / \mathrm{m}$ \\
\hline \hline
\end{tabular}

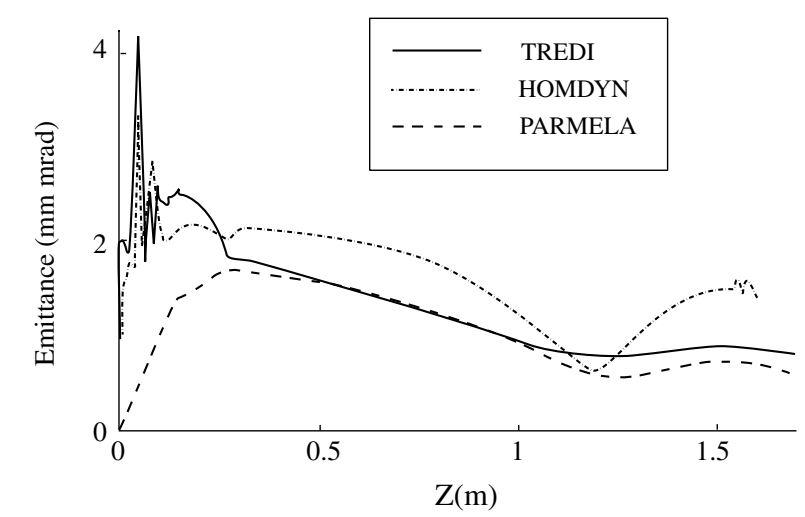

FIG. 6. Comparison between TREDI, HOMDYN, and PARMELA with the parameters listed in Table II. The radial emittance is plotted for the first $1.70 \mathrm{~m}$ along the beam line.

charge $10 \mathrm{ps}$ pulse. The simulations have shown a good agreement between all the codes, except for a shift of the envelope minimum in the drift out of the gun and a slight difference for the overall energy spread given by HOMDYN.

\section{CODES FOR BUNCH COMPRESSOR DESIGN}

The highest normalized beam brightness obtained in a $\mathrm{rf}$ photoinjector is of the order of $10^{14}[33,34]$, while the beam brightness required in the design parameters of both LCLS [35] and TTF [36] SASE FELs projects is approximately $4 \times 10^{15}$. Although an improvement in the injector brightness at the injector output is expected with the introduction of $\mathrm{rf}$ compression [37,38], the remaining gap should be filled with an increase of the normalized beam brightness obtained by a magnetic beam compression. The scheme is the following: a correlated energy slope is induced by an off crest operation of the accelerating $\mathrm{rf}$ field. This correlation is exploited in a dispersive magnetic chicane to produce longitudinal focusing. One of the most challenging issues related to longitudinal compression is due to the coherent spontaneous radiation produced by the electrons in the tail of the bunch that reaches the head along the bend and interacts with the bunch itself. We have a broad-band longitudinal and transverse coupling whose main effect is that of inducing an energy spread that is transformed into an emittance increase by the longitudinal-to-transverse coupling of the chicane itself. We may distinguish two different regimes, a low frequency regime where the coherent spontaneous emission produces a correlated energy spread over the whole bunch [39], and a higher frequency regime where the microbunching instability [40-43] induces the growth of uncorrelated energy spread and emittances. This latter process resembles that of a free-electron laser with a broad-band gain associated with the chicane magnetic field $[42,43]$. The peak frequency of the feedback gain is located at a few tens of microns a typical scale length which is much shorter than the bunch length itself. 
It is indeed this high frequency broad-band coupling that creates the most difficulties from the simulations point of view. We may distinguish two different approaches in the simulation of the processes falling under the name of CSR effects. In the first case the codes have the typical structure of TREDI and are based on first principles. The algorithm consists of evaluating the interaction between the macroparticles according to the Lienard-Wiechert retarded potentials, building a field representation over the particles positions, and advancing the particles of a time step. The second approach is based on the "line charge method," i.e., based on the expression of the longitudinal wake of a line charge distribution moving in a curved path [39].

\section{A. Simulation of CSR effects}

While TREDI has been developed for the simulation of linacs and photoinjector dynamics, other codes such as TRAFIC $^{4}[44,45]$ developed by Dohlus, Limberg, and Kabel, and a "not named" Li code [46] (will be identified as "RL" in the following) have been specifically designed for the simulation of CSR effects. Both are based on first principles with noise suppression obtained by giving a finite size to macroparticles. The procedure adopted for the numerical noise suppression is the main difficulty and the main source of differences between the predictions of this class of codes. The relatively high frequencies involved require a large number of macroparticles, especially for the simulation of the microbunching instability, while the implementation of retarded effects and the preservation of causality in the evaluation of the integral over a finite dimension charge distribution is time consuming and severely limits the number of macroparticles that can be practically simulated. According to the example of a Gaussian bunch given in the introduction (see Fig. 1), the microbunching gain spectrum falls within the spectral region of amplitude $1 / \sqrt{N}$, where $N$ is the number of macroparticles. This process is seeded by a numerical shot noise and the seed amplitude scales unfavorably with the root of the number of macroparticles. A possibility to overcome this limitation is offered by the "pseudorandom" number sequences [47]. These sequences of apparently only random numbers have the characteristic of filling the initial space more uniformly than random numbers and producing reduced fluctuations whose amplitudes scale with $1 / N$ in place of $1 / \sqrt{N}$. This quiet start method is implemented in ELEGANT and TREDI with the Sobol sequences [47]. The noise reduction obtained with pseudorandom sequences allows the simulation with a lower number of macroparticles and constitutes a significant advantage in three-dimensional simulations of the rf-gun dynamics. In a "start to end" simulation, the phase space variables are passed from an injector/linac devoted code to a CSR simulation code. For this reason pseudorandom generators are also implemented in the generation of the initial phase space in PARMELA and ASTRA simu- lations which are then used, e.g., as input in TRAFIC ${ }^{4}$ simulations. Unfortunately the preservation of this condition of reduced fluctuations of the phase space along the dynamics remains an open problem. The beam modifications induced by nonlinear processes alter the uniformity characteristics of the initial beam distribution and larger fluctuations appear. The target of executing simulations with a large number of particles remains fundamental. For this reason the RL code is limited to two spatial dimensions in the orbit plane. In TRAFIC ${ }^{4}$ the beam has a twofold representation. It is represented as a continuous charge distribution when it is considered as a source of the electromagnetic fields, and an ensemble of pointlike particles when the effects of the fields are evaluated. The beam line is divided in slices and the "field generating" bunch is propagated along the beam line. The beam parameters along the beam line are calculated and stored. The field on "pointlike" particles following the beam line is then evaluated according to the retarded conditions and the information on the dynamics of the pointlike particles is then used to advance a new field generating bunch along the beam line. The iteration of this procedure leads to the self-consistency of the method. TRAFIC ${ }^{4}$ is capable of handling also boundary conditions of infinitely extended, perfectly conducting flat walls by the image charge method. The computation time grows in this case and a few thousand particles require several days of CPU time, depending on the chicane complexity and length. A parallel implementation of the code is practically a must in the simulation of these cases [48].

An alternative for the solution of this problem is the line charge method. Several simulation codes are indeed based on the formulation of the wake potential due to a line charge distribution following an arc [39]. Expressions for the potential of the type

$$
\begin{array}{r}
\frac{d E(s, \phi)}{d(c t)}=\frac{-2 e^{2}}{\left(3 R^{2}\right)^{1 / 3}}\left\{s_{l}^{-1 / 3}\left[\left(s-s_{l}\right)-\lambda\left(s-4 s_{l}\right)\right]\right. \\
\left.+\int_{s-s_{l}}^{s} \frac{1}{(s-z)^{1 / 3}} \frac{d \lambda(z)}{d z} d z\right\}
\end{array}
$$

which holds for a generic longitudinal charge distribution $\lambda$ entering in a bending magnet with bending radius $R$ and bending angle $\phi$, and where $s_{l}=\left(R \phi^{3} / 24\right)$ is the slippage length defined as the path difference of the curved trajectory of the charged particle in the magnet and the straight line followed by the light, have been derived also for a bunch in steady state circular motion and exiting the bending. The formulation has been extended by Stupakov and Emma to include the postdipole region and is the basis for the CSR implementation in ELEGANT [49,50], CSR_CALC (Emma [51]), and the Dohlus line charge program [52]. The main assumptions are the following:

(i) The electron motion is assumed ultrarelativistic. 


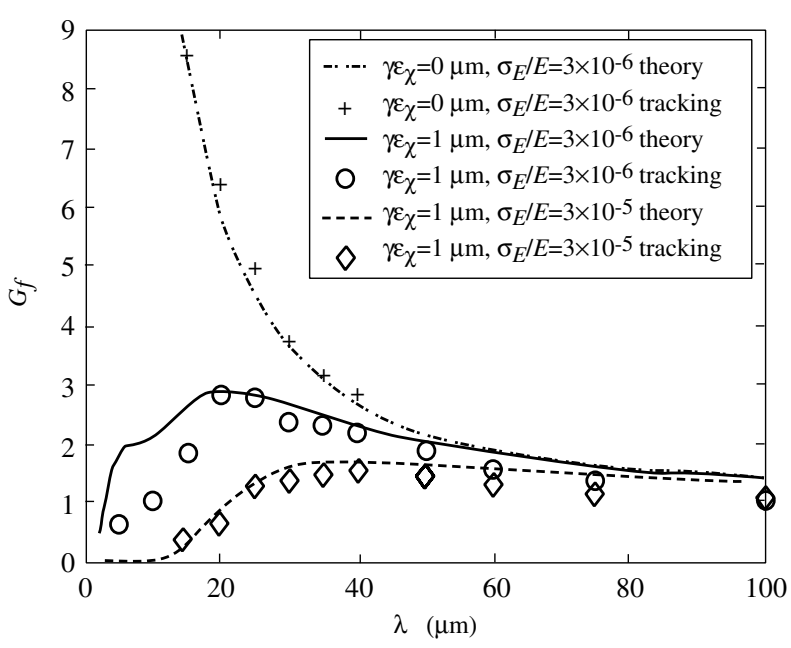

FIG. 7. Gain spectrum of microbunching modulation in the SLAC BC2 compressor at different input energy spread and emittances.

(ii) A one-dimensional beam is assumed for CSR field calculations. The "real" beam is projected along the curvilinear coordinate $s$.

(iii) Transverse internal forces are neglected.

(iv) Nondissipative interaction forces are not considered. Only radiative terms are retained.

(v) Any change in the charge distribution at retarded time is neglected; the wake is calculated assuming that the charge distribution has not changed according to compression.

The advantage in terms of computational time is consistent. In Fig. 7 is shown a plot of the microbunching gain in the LCLS BC2 compressor [53] as a function of the initial modulation wavelength (prior to compression). The agreement with theory (continuous lines) $[41,42]$ is remarkable. It is even more remarkable in the fact that the simulation, done with $2 \times 10^{6}$ particles, finished in less than $1 \mathrm{~h}$ on a $1 \mathrm{GHz}$ PC [54].

The possibility of running such a large number of macroparticles allows the simulation of microbunching effects with a reasonably low numerical noise.

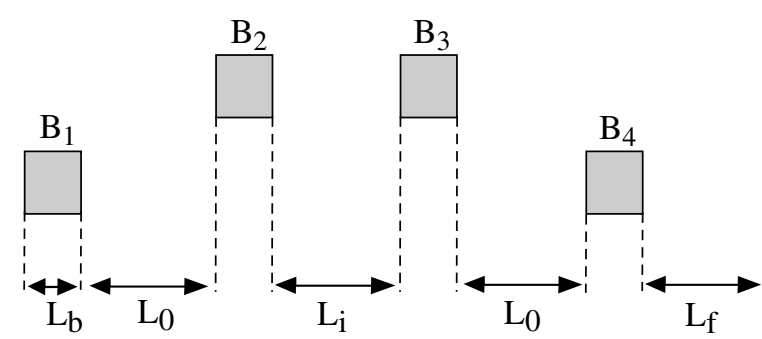

FIG. 8. Layout of the four bends test chicane considered for code benchmarking.

\section{B. Comparison between CSR devoted codes}

A comparison between the results predicted by the various mentioned programs has been obtained on a test case based on a four bends magnetic chicane on occasion of the ICFA Beam Dynamics mini workshop [55]. In Fig. 8 is shown the layout of the four bend chicane which served as a test case, with the parameters listed in Table III.

The simulations were performed at electron beam energies of 0.5 and $5 \mathrm{GeV}$ with electron pulse shapes both Gaussian and stepwise, but a complete comparison between all codes was available only at $5 \mathrm{GeV}$ with a Gaussian longitudinal current profile. The other electron beam parameters for the simulation are shown in Table IV. The results of the comparison relevant to the Gaussian distribution are summarized in Table $\mathrm{V}$, where the relative energy loss $(\delta E)$, the energy spread variation $\left(\delta \sigma_{E}\right)$, and the normalized emittance in the orbit plane are given after the chicane. The simulations, except the one obtained with TREDI [4], have shown increments of the normalized emittance between $30 \%$ and $50 \%$. The codes based on the line charge model have provided similar results, showing a reasonable relative agreement. The output from "first principles codes" is more scattered, reflecting the existing difficulties in terms of noise suppression and "resolution," which is limited by the complexity of the integration schemes and by the consequent low number of particles that can be simulated. The simulation done with TREDI was obtained with only

TABLE III. List of the four bends test chicane parameters.

\begin{tabular}{lcc}
\hline \hline Chicane Parameters & Symbol & Value \\
\hline Bend magnet length (projected) & $L_{b}$ & $0.5 \mathrm{~m}$ \\
Drift length $B 1 \rightarrow B 2$ and $B 3 \rightarrow B 4$ (projected) & $L_{0}$ & $5.0 \mathrm{~m}$ \\
Drift length $B 2 \rightarrow B 3$ & $L_{i}$ & $1.0 \mathrm{~m}$ \\
Post chicane drift & $L_{f}$ & $2.0 \mathrm{~m}$ \\
Bend radius of each dipole magnet & $R$ & $10.35 \mathrm{~m}$ \\
Bending angle & $F$ & $2.77^{\circ}$ \\
Momentum compaction & $R 56$ & -25 \\
2nd order momentum compaction & $T 566$ & $+37.5 \mathrm{~mm}$ \\
Total projected length of chicane & $L_{\mathrm{TOT}}$ & $13.0 \mathrm{~m}$ \\
\hline \hline
\end{tabular}


TABLE IV. $\quad e$-beam parameters used in the benchmark.

\begin{tabular}{lcc}
\hline \hline Electron beam parameters & Symbol & Value \\
\hline Nominal energy & $E$ & $5 \mathrm{GeV}$ \\
Bunch charge & $Q$ & $1.0 \mathrm{nC}$ \\
Incoherent energy spread & $\Delta E$ & $10 \mathrm{keV}$ \\
Linear energy - z correlation & $A$ & $36 \mathrm{~m}^{-1}$ \\
Initial rms energy spread & $\Delta E / E$ & $0.72 \%$ \\
Initial rms bunch length & $\sigma_{i}$ & $200 \mu \mathrm{m}$ \\
Final rms bunch length & $\sigma_{f}$ & $20 \mu \mathrm{m}$ \\
Initial normalized rms emittances & $\varepsilon_{x} / \varepsilon_{y}$ & $1.0 / 1.0 \mathrm{~mm} \mathrm{mrad}$ \\
Initial betatron functions at first bend & $\beta_{x} / \beta_{y}$ & $40 / 13 \mathrm{~m}$ \\
Initial alpha functions at first bend entrance & $\alpha_{x} / \alpha_{y}$ & $+2.6 /+1$ \\
\hline \hline
\end{tabular}

TABLE V. List of benchmarked codes and of the beam parameters at the end of the chicane. We have indicated with $\delta E$ the relative energy loss and with $\delta \sigma_{E}$ the change in the relative energy spread.

\begin{tabular}{lcccc}
\hline \hline Dimension & Code Name & $\delta E(\%)$ & $\delta \sigma_{E}(\%)$ & $\varepsilon$ \\
\hline 3D & TRAFIC4 & -0.058 & -0.002 & 1.4 \\
3D & TREDI & -0.041 & 0.017 & 2.3 \\
2D & Program by Li & -0.056 & -0.006 & 1.32 \\
1D line charge & ELEGANT & -0.045 & -0.0043 & 1.55 \\
1D line charge & CSR_CALC (Emma) & -0.043 & -0.004 & 1.52 \\
1D line charge & Program by Dohlus & -0.045 & -0.011 & 1.62 \\
\hline \hline
\end{tabular}

$10^{3}$ macroparticles and was affected by a strong numerical noise. Part of the noise was suppressed by discarding $15 \%$ of the "worse" macroparticles. TREDI simulations done after the workshop with up to $10^{4}$ macroparticles [56] have shown results similar to the ones obtained with the other programs, with an emittance growth of about $70 \%$ and with an energy loss along the chicane of about $0.02 \%$. In terms of accuracy of the results, the convergence of the data provided by the line charge codes shows at least that the line charge model is in general correctly implemented. A margin for improvement exists in the convergence of the predictions of first principles codes that would give more reliability on the absolute correctness of the models.

\section{CODES FOR FREE-ELECTRON LASER DESIGN}

The simulation of SASE free-electron lasers has been obtained both with PIC codes [8] and with LienardWiechert based algorithms [57,58]. These methods, that have the merit of allowing a verification of FEL physics formulation from first principles, have been applied only to a limited number of specific cases. They indeed do not take any advantage of the fact that in a free-electron laser the electron beam interacts with the periodic magnetic field of the undulator and with the optical field of a copropagating electromagnetic wave with a $k$ vector ori- ented in the $z$ direction. The FEL "instability" is characterized by a gain with a narrow bandwidth of the order of the Pierce parameter $\rho$ [5,59],

$$
\rho=\frac{1}{4 \pi \gamma}\left[\frac{2 \pi^{2}}{\sum_{e}} \frac{I_{\text {peak }}}{I_{A}}\left[K f_{B}(\xi) \lambda_{u}\right]^{2}\right]^{1 / 3},
$$

where

$$
f_{B}(\xi)=J_{0}(\xi)-J_{1}(\xi), \quad \text { and where } \xi=\frac{1}{4} \frac{K^{2}}{1+\frac{K^{2}}{2}} .
$$

$\lambda_{u}$ is the undulator period, $\gamma$ the relativistic factor, $K=$ $e B_{\text {peak }} \lambda_{u} / 2 \pi m c$ the undulator strength, $I_{\text {peak }} / I_{A}$ is the ratio between the peak current and the Alfvèn current $(17040 \mathrm{~A})$, and $\Sigma_{e}$ is the transverse electron beam cross section. Typically, in the case of short wavelength FELs, the Pierce parameter is smaller than $10^{-2}$. For this reason almost all the codes devoted to FELs are based on the paraxial wave approximation (i.e., $k=k_{z}$ ) and on the slow varying envelope approximation (SVEA), which consists of assuming that the field may be written as the product of a term oscillating at the resonant frequency of the instability and propagating in the $z$ direction, and a slowly varying complex amplitude

$$
\vec{E}(z, \vec{r}, t)=\left(a(z, \vec{r}, t) \exp \left(i\left(k_{Z} z-\omega_{L} t\right)\right) \quad 0 \quad 0\right)
$$

where the function $a(z, \vec{r}, t)$ satisfies the following 
inequalities:

$$
\left|\frac{\partial^{2} a}{\partial t^{2}}\right| \ll \omega_{L}^{2} a, \quad \text { and } \quad\left|\frac{\partial^{2} a}{\partial z^{2}}\right| \ll k_{L}^{2} a .
$$

This assumption leads to a significant simplification of the wave equation. The second derivatives of $a$ in Maxwell's equations may be neglected and the wave equation for the field reduces to

$$
\left[\frac{\partial}{\partial z}+\frac{1}{c} \frac{\partial}{\partial t}-\frac{i}{2} \nabla_{\perp}^{2}\right] a(z, \vec{r}, t)=-\left.J(z, \vec{r}, t)\left\langle\frac{e^{-i \zeta_{i}}}{\gamma_{i}}\right\rangle_{\{i\}}\right|_{(z, r, t)},
$$

where the function $J(\vec{r}, z, t)$ is a generalized beam current that contains the macroscopic dependence of the $e$-bunch profile on coordinates and time. The microscopic contribution to the transverse current is contained on the average of over the electrons phases $\zeta_{i}=\left(k_{L}+k_{u}\right) z_{i}-\omega_{L} t$ for $\left\{i=1, n_{e}\right\}$ and with $k_{u}=2 \pi / \lambda_{u}$ on the right-hand side (rhs) of (23), which is a "slow" function of position and time. The average is indeed calculated on a scale length which is larger than (or equal to) the laser wavelength, but is smaller than the distance over which the function $a$, satisfying Eq. (22), has significant changes. Equation (23) depends only on slowly varying functions. The transformation to a fields description based on slowly varying variables allows a drastic reduction of the mesh requirements and the solution of the Maxwell's equations in the form (23) may be numerically obtained with a partial differential equation solver. This is done assuming axial symmetry in TDA [60] and in GINGER [61,62] or in three dimensions, in TDA3D [63] with a field decomposition into cylindrical modes, and in Cartesian geometry in FELEX/N [64,65], and in GENESIS [66] using an alternating direction implicit integration scheme [7]. In MEDUSA $[67,68]$ the source dependent expansion technique has been implemented $[69,70]$. This technique consists of the expansion of the field $a(z, \vec{r}, t)$ in terms of GaussHermite functions with characteristic parameters depending on the source term $J(z, \vec{r}, t)$. The expansion allows an efficient solution of the wave equation, representing the field with a reduced number of eigenfunctions.

The electron dynamics in MEDUSA is integrated by directly solving the Lorentz force equations with a fourth order Runge-Kutta [7], with the fields given by the superposition of the laser and undulator fields, respectively,

$$
m \frac{d(\gamma \vec{\nu})}{d t}=-e\left(\vec{E}+\frac{\vec{v}}{c} \times \vec{B}\right), \quad \frac{d \gamma}{d t}=-\frac{e}{m c^{2}} \vec{E} \cdot \vec{\nu} .
$$

This "first principles" approach allows the possibility of accepting 3D field maps which includes the undulators and all additional field components (i.e., F0D0 lattices, etc.). The integration of the 3D Lorentz force equations facilitates the adaptation of MEDUSA to treat novel magnetic field configurations and beam distributions as, e.g., in the case of biharmonic undulators [71]. The drawback is that MEDUSA does not take advantage of the periodicity of the undulator magnetic fields, and several tens of Runge-Kutta steps are required for the correct integration of the trajectory along a single undulator period. Most of the other FEL devoted codes (as, e.g., GINGER, FELEXN, PROMETEO [72]) use the Kroll, Morton, and Rosenbluth (KMR) approximation [73], consisting of assuming that the wiggling amplitude is small and that the frequency of any variation of the parameters entering in the FEL process is large with respect to the frequency of oscillation due to the undulator magnetic field, i.e.,

(i) adiabatic change of undulator parameters as, e.g., magnetic errors, tapering,

$$
\frac{1}{\lambda_{u}} \frac{d \lambda_{u}}{d z} \ll 1, \quad \frac{1}{\lambda_{u}} \frac{d K}{d z} \ll 1
$$

(ii) The scale of variation of the $e$-beam parameters is large, i.e., the betatron period $\lambda_{\beta}$ is much larger than the undulator period $\lambda_{\beta} \gg \lambda_{u}$ and changes in current, energy, etc. occur on a scale of many undulator periods.

Under these assumptions the particle trajectory is averaged over the undulator period and the required time step length may be larger than the undulator period itself consisting of a significant advantage from the CPU time point of view. When the effects of the laser field on the electron transverse motion can be neglected, the equations of motion can be furthermore simplified (as, e.g., in GENESIS). In the specific case of a Linear undulator with strength $K=K(\vec{r})$, we have

$$
\begin{aligned}
\frac{d \gamma}{d t} & =-c k_{L} \frac{f_{B}(\xi) K(\vec{r})}{\sqrt{2} \gamma}|a(z, \vec{r}, t)| \sin (\zeta+\phi(z, \vec{r}, t)), \\
\frac{d \zeta}{d t} & =c k_{u}-c k_{L} \frac{1+\left|\vec{p}_{\perp}\right|^{2}+\frac{K(\vec{r})^{2}}{2}-\sqrt{2} K(\vec{r})|a(z, \vec{r}, t)| \cos (\zeta+\phi(z, \vec{r}, t))}{2 \gamma^{2}}, \quad \frac{d \vec{p}_{\perp}}{d t}=-\frac{c}{4 \gamma} \frac{\partial K(\vec{r})^{2}}{\partial \vec{r}}+c \vec{k}_{\mathrm{ext}} \vec{r}, \\
\frac{d \vec{r}}{d t} & =c \frac{\vec{p}_{\perp}}{\gamma},
\end{aligned}
$$

where $\phi(z, \vec{r}, t)=\arg (a(z, \vec{r}, t))$ is the slowly varying phase of the laser field and $\vec{k}_{\text {ext }}$ is the focusing strength relative to additional focusing elements external to the undulator. The characteristic Colson's pendulumlike equation [74] is readily derived from Eq. (26) by neglecting the transverse dynamics terms and the term proportional to $k_{u}$ on the rhs of the 
second equation of (26). This assumption is very well satisfied for FELs operating with high beam energy and short wavelength $\lambda_{u} \gg \lambda_{L}$. The FEL pendulum equation in the conjugate variables, $\zeta, \nu=d \zeta / \tau$, with $\tau=$ $\beta_{z} c t / L_{u}$ is the equation of motion corresponding to the Hamiltonian

$$
H=\frac{1}{2} \nu^{2}-a \cos (\zeta)
$$

with the assumption of a steady state, uniform $e$-beam distribution; the field $a$ defined in Eq. (21) is dependent on $(\tau)$ only. Both the coupled electron equations of motion derived from the Hamiltonian Eq. (27) and the field Eq. (23) are periodic in $\zeta$, with period $2 \pi$. The solution in this 0 -dimensional case is obtained by imposing periodic boundary conditions to particles and fields [75]. The solution of this simple case may be used as the basic element for the extension to higher dimensional cases.

\section{A. Quiet start and modeling of the shot noise}

The approximations for fields (SVEA) and particles (KMR) can be considered as mutually consistent assumptions. With the KMR formulation we neglect wide bandwidth components in the field sources dynamics, while with the SVEA approximation we assume that the FEL gain is nonzero in the same narrow frequency window centered around the lasing frequency $\omega_{L}=2 \pi c / \lambda_{L}$. The width of this window is proportional to the parameter $\rho$ defined in Eq. (19). The numerical representation of the electron beam spectral distribution must resemble that of the real distribution in this frequency interval. Randomly distributed macroparticles, unless their number is comparable to the real number of electrons in the bunch, provide a distribution with a much higher spectral content than the physical one. The correct noise level is introduced by exploiting the periodicity of the Hamiltonian (27). The beam is indeed represented as an ensemble of "beamlets," each one with macroparticles distributed as

$$
\zeta_{i}=\zeta_{0}+i \frac{2 \pi}{n_{p}}+\delta_{i}, \quad i=0,1, \ldots, n_{p}-1,
$$

where the factors $\delta_{i}$ are randomly distributed coefficients used to introduce an artificial shot noise. At $\delta_{i}=0$ the harmonic content of such a beamlet is exactly zero at the fundamental wavelength and at the higher order harmonics $h=2, \ldots, n_{p}-1$ (see Fig. 9). The number of macroparticles per beamlet should be larger than the corresponding number suggested by the simple application of the Nyquist theorem. With the definition (28), at the beginning the particles are equally spaced and $2 h$ particles would be adequate for a noiseless quiet start of the $h$ harmonic. When the dynamics is applied, the condition provided by the Nyquist theorem does not hold anymore. First, the FEL couples bunching at a given harmonic $h$ with the field growth at harmonics $h \pm 1$

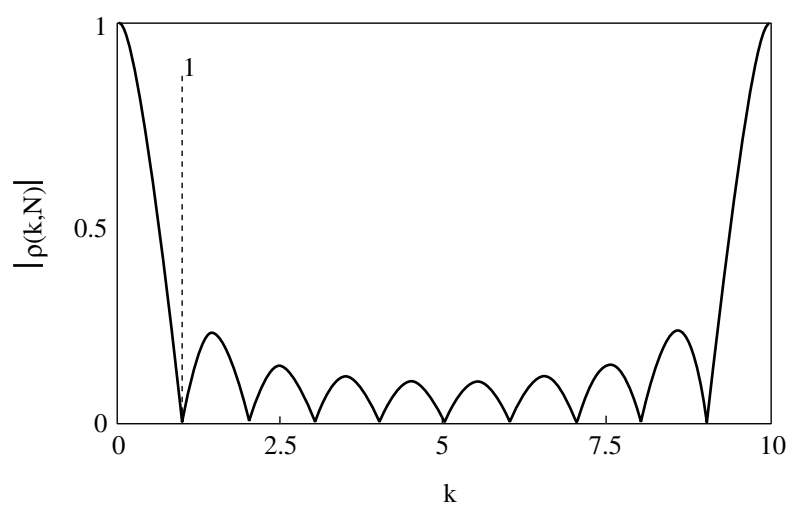

FIG. 9. Fourier transform of a beamlet composed of ten macroparticles. The spectral content of the distribution is zero at $k=1.9$.

[76]. Second, and this is even more critical, the particles positions are shifted from equally spaced positions, and a bunching contribution due to an insufficient original sampling frequency, especially at the highest harmonics, appears. At saturation the macroparticles are bunched, and phase space discontinuities in the lower density regions strongly affect the bunching estimation. This is particularly critical for the higher order harmonics.

The effect of shot noise is introduced with the shifting factors $\delta_{i}$. A shot noise of amplitude proportional to the average macroparticles displacement is obtained. An algorithm to produce these displacements with the correct noise statistics in a $1 \mathrm{D}$ case is given by Penman and McNeil [77]. Particular care must be taken in the generation of beamlets in multidimensional spaces to avoid undesired interferences that may arise from the dynamical effects (drifts, betatron motion etc.). An elegant description of the shot noise implementation in GINGER and the problems related to the generalization to multidimensional spaces are given in Ref. [78].

\section{B. Time dependent simulations}

At the resonant wavelength the radiation slips over the electron bunch of one optical wavelength per undulator period. After the propagation in an undulator of $N$ periods, the radiation passes over a portion of length $N \lambda_{L}$ of the electron bunch. The "slippage length" is the name of this causally connected region of length $N \lambda_{L}$. For typical numbers, in the case of short wavelength FELs, the slippage length is shorter than the electron bunch length. The radiation field is not propagating in free space, but in a gain medium (the electron beam) that is exponentially amplifying the radiation. As a consequence the phase information of the radiation is "lost" after the propagation over the distance of the order of $\lambda_{L} / 4 \pi \rho$ along the electron bunch, which is commonly indicated as the "cooperation length" and is shorter than the slippage length if the FEL reaches saturation. For the same 
reason the relative gain bandwidth is proportional to $\rho$. In the implementation of time dependent simulations in GINGER and in GENESIS a time window larger than a cooperation length, that is enclosing the whole electron beam or part of it, is defined. This time window is then sampled and sliced as shown in Fig. 10. The slices have a length of one optical wavelength (one of the beamlets defined in the previous section) and are separated by a distance $\Delta$. In GINGER the simulation evolves at discrete time steps of length $\Delta \lambda_{u} /\left(\lambda_{L} c\right)$ and at each time step the field relevant to one slice is shifted to the next slice. In GENESIS the simulation for the first slice is done up to the end of the undulator, and then the values of the fields are used to execute the simulation for the second slice. The procedure is repeated up to the end of all the slices. This procedure allows one to keep in memory only one slice at a time, but from the conceptual point of view it is essentially the same as that adopted in GINGER. The parameter $\Delta$ must satisfy some constraints. According to the Nyquist theorem the cutoff frequency is given by twice the sampling frequency, which is $1 / \Delta$.

Imposing the condition that the band pass associated with the sampling procedure must be larger than the FEL gain bandwidth, we find the condition

$$
\Delta_{\max } \ll \frac{\lambda_{L}}{4 \pi \rho} .
$$

In general the simulation is done with $\Delta<\Delta_{\max } / 8$ to ensure that the whole gain bandwidth is contained in the numerical representation. The simulation time window should be larger than a slippage length. In GENESIS, when only a portion of the beam is simulated, the first slippage length may be ignored in the output, as it is used as the source of the field for the following slices. It is obviously retained in a simulation enclosing the whole beam. The simulation of long bunches is obtained in GINGER by assuming steady state parameters for the electron beam distribution along the bunch and by setting a periodicity condition on the time window.

\section{Codes validation}

The output of these codes provides much information on the saturation length and other characteristics of the

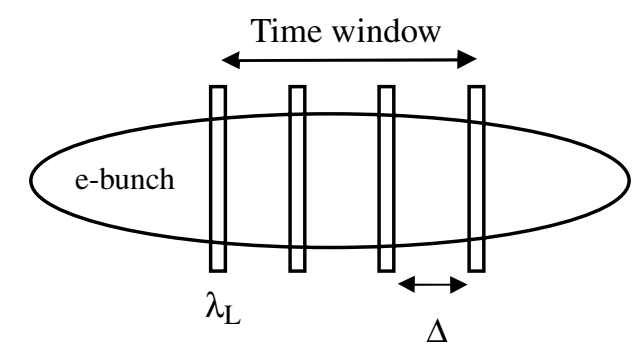

FIG. 10. Slicing of the $e$ bunch for time dependent simulations.
FEL, as sensitivity to $e$-beam parameters, stability, spectrum, pulse duration, coherence, etc. There are a number of theoretical approaches that allow one to obtain analytic estimates of some of these features [79-82]. These analytical formulations are useful in narrowing the range of the input parameters for the simulation, but specific effects such as, e.g., magnetic errors or alignment errors or the effects of specific distributions as, e.g., a folding of the input 6D electrons phase space, are conveniently analyzed with the numerical approach. For this reason the codes are widely used in the design of future sources and the ongoing interest in their validation against the available experimental data and a cross comparison of their results are justified. The program MEDUSA has been extensively used in the prediction and the analysis of the LEUTL experiment [83,84]. In Ref. [84] the most known multidimensional FEL codes have been compared on LEUTL parameters. The results have shown, in a steady state mode, resonant wavelengths within $0.2 \%$ and saturation lengths within $10 \%$. The differences between the codes increased in the simulation of a multisegmented undulator with MEDUSA providing the largest saturation length. The simulation of the LEUTL device is affected by strong diffraction effects which become more pronounced in the gaps between the undulators, where the lack of gain guiding produces a rapid expansion of the laser mode. The observed difference could be justified by the different representation of the transverse fields, obtained with an expansion on free space modes in MEDUSA, and sampled on a mesh, in the other mentioned codes. The agreement should be however considered satisfactory, on the light of the quoted differences in the models, in the algorithms, and the quite large number of parameters entering in these simulations. The VISA $800 \mathrm{~nm}$ FEL experiment has been reproduced in surprisingly good detail with GENESIS $[85,86]$.

\section{Harmonics}

When a SASE FEL approaches saturation, the modulation of the longitudinal electron beam distribution develops substantial Fourier components at higher harmonics of the fundamental and emission at the corresponding wavelengths occurs. There is widespread interest in this process, because emission on high order harmonics represents a significant resource to extend the wavelength tunability of a free-electron laser [87-95]. We may distinguish two different cases:

(1) when the dynamics is driven by the fundamental and the harmonic emission is a byproduct of the phase space modulation.

(2) When the electron dynamics is self-consistently modified by the radiation field of the harmonics.

In the first case the harmonics emission depends on the histories of particles along the undulator. The bunching coefficients, i.e., the Fourier components of the longitu- 
dinal electron beam distribution depending on the macroparticles longitudinal coordinates $\zeta_{i}$

$$
b_{h}=\left\langle e^{-i h \zeta_{i}}\right\rangle_{\{i\}}
$$

and the corresponding harmonic emission may be calculated in the postprocessing phase. The main required precaution consists of a correct preparation of the beam quiet start at the desired harmonic. This method is used both in GINGER and in GENESIS. FELEXN has the capability of operating at higher harmonics, but no coupling between the harmonics is included.

In MEDUSA, PROMETEO, and in PERSEO (1D, time dependent [75]) the harmonics are treated self-consistently, by solving the particle dynamics with the fields given by the superposition of the fundamental and of the higher order harmonics. This approach is the most appropriate in the simulation of exotic undulator configurations [71] that were proposed as tools to enhance the harmonic emission in SASE FELs. The increased harmonic intensity in saturated conditions is indeed large enough to affect the electrons phase space trajectories and to show a different behavior than the one expected in a single harmonic simulation. An example of this effect is given in Fig. 11, where a snapshot of the longitudinal phase space obtained in a 1D simulation with PERSEO is shown. The undulator considered is of a "biharmonic" type [71], with main period $\lambda_{u}=6 \mathrm{~cm}$ and strength $K=1.4$. The enhance-

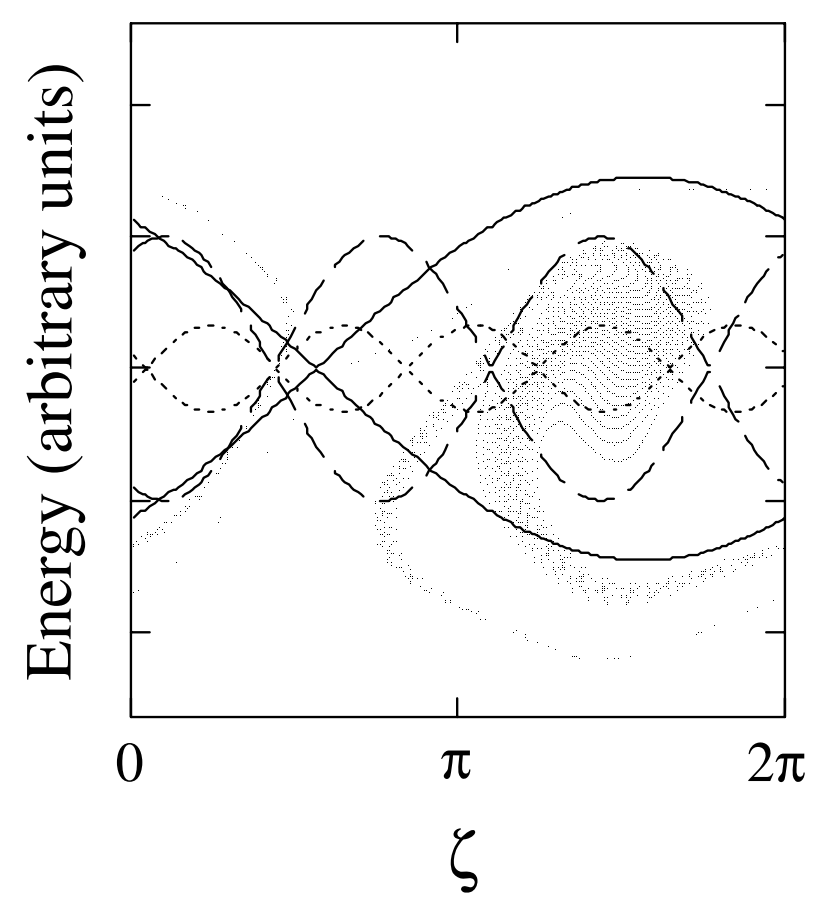

FIG. 11. Longitudinal $e$-beam phase space (energy phase) in a free-electron laser operating with a biharmonic undulator, at saturation. The separatrices corresponding to the fundamental (continuous line) to the 3rd (dashed line) and to the 5th harmonic fields are shown. ment of the coupling on the third harmonic is obtained by superimposing a second undulator, with the field oriented in the orthogonal direction, with the same strength $K$ and period $\lambda_{u, 3}=\lambda_{u} / 3=2 \mathrm{~cm}$. The beam energy considered in this example is $1 \mathrm{GeV}$, which defines the resonance at a wavelength of about $20 \mathrm{~nm}$. The peak current is $140 \mathrm{~A}$ with $1 \mathrm{~mm}$ mrad normalized emittance and a relative energy spread of $0.5 \times 10^{-3}$. The phase space image of Fig. 11 is taken at $z \cong 40 \mathrm{~m}$, at saturation. The separatrix corresponding to the third harmonic (dashed line) is wide enough to trap a large fraction of the macroparticles and to modify the longitudinal beam dynamics. This behavior, which affects both the energy conversion efficiency into higher harmonics and the spectral features of the radiated fields, is mainly relevant in conditions of enhanced harmonic emission, as those realized with biharmonic undulators. In this respect it should be investigated in time dependent mode and with 3D codes including the harmonic field in the dynamics, as MEDUSA, to understand the implications on the spatial and temporal coherence of the emitted field. MEDUSA has been extensively used [96] in the prediction of the performances on higher order harmonics of the LEUTL experiment. Comparisons between the predictions of MEDUSA, PROMETEO, and GINGER in conditions similar to those of the LCLS FEL and of LEUTL FEL are given in Refs. [97,98] respectively. The sensitivity of the harmonic generation process to the beam quality has been analyzed. In both cases the electron's longitudinal dynamics and the harmonic emission are mainly driven by the laser field on the fundamental harmonic. For this reason the comparison was not apparently affected by the lack of self-consistency on the harmonic fields of GINGER.

\section{CONCLUSIONS}

There are several other sources of intrabeam coupling and nonlinear collective effects that play an important role in the design of short wavelength free-electron lasers, as wake field effects, beam loading, etc. The three regimes analyzed in this review: beam generation, beam compression, and the FEL process were characterized by quite well-established families of investigation tools devoted to the simulation of the specific regimes. We had the opportunity to analyze, from the spectral point of view, the constraints posed by the numerical representation, which is the main reason why, in the considered conditions, the simulation of charged particles interacting with electromagnetic field cannot be accomplished by a single code. In general we may distinguish between codes based on first principles and codes based on semianalytic models. The first include most of the physical aspects of the problem. This completeness is usually paid in terms of complexity of the implementation and in terms of calculation time. In the second case the numerical implementation follows a theoretical approach which, according to 
some physical approximations, allows one to point to some specific relevant issue with a fast relaxation of the usually large number of parameters involved. An example is provided by the line charge method in the calculation of the CSR microbunching. The number of particles simulated with this method is large enough to reduce to an acceptable level the numerical noise in the wide band spectrum of the CSR impedance. The approximations considered may be quite stringent, but the large number of macroparticles allows a much better representation of the beam statistics than the one obtainable with a first principle approach. In general these two families of codes can be considered both necessary and complementary.

The modeling aspects of the codes have been privileged, but several other features have great importance. An example is provided by the interface. The amount of data produced when $6 \mathrm{D}$ phase spaces with $10^{4} / 10^{6}$ macroparticles are considered, is huge. The interface is meant not only in terms of a simple human interface for the analysis and visualization of the results. The necessity of different codes handling different regimes means that the exchange of phase spaces in a compatible format between different programs is a practical, but still essential problem. Significant effort in this direction has been done with the SDDS [99] and HDF5 [100] data exchange format. Several examples of start to end simulations can be found in the literature [101,102].

The last, but not least, aspect is the documentation and the sources availability. The sources availability is of great importance for the peer review possibility offered when the user is not faced by a "black box," but has the opportunity to analyze directly the sources and the equation he is dealing with. When the documentation or/and sources were available, we referenced the link to the web pages where this information can be retrieved from the network.

\section{ACKNOWLEDGMENTS}

I am pleased to acknowledge the contribution of Marcello Quattromini for many useful discussions on various aspects of the fields regularization problem and for the patience shown in the development and execution of TREDI. I express my gratitude to Pino Dattoli for his support in preparing this manuscript and for the numerous discussions we had on various aspects of the FEL physics. I wish to acknowledge the contributions of Riccardo Bartolini, Luca Mezi, Massimo Ferrario, Jamie Rosenzweig, Luca Serafini, and Titti Ronsivalle for many useful discussions, suggestions, and for providing some of their results. I also gratefully acknowledge the contributions of Sandra Biedron, Michael Borland, Martin Dohlus, Paul Emma, Bart Faatz, William Fawley, Klaus Floettmann, Henry Freund, Andreas Kabel, John Lewellen, Torsen Limberg, Cecile Limborg, Steven Milton, Sven Reiche, and Rui Li for their solicitude in providing information, references, and viewgraphs about their work.

[1] J. M. Dawson and A.T. Lin, in Handbook of Plasma Physics, edited by M. N. Rosenbluth and R. Z. Sagdeev, Basic Plasma Physics Vol. 2 (Elsevier Science Publisher, New York, 1984).

[2] T. P. Wangler, LANL Report No. LA-UR-93-805, 1993.

[3] M. Quattromini, in Proceedings of the 2nd Melfi School on Advanced Topics in Mathematics and Physics, Melfi, 2000, edited by G. Dattoli, H. M. Srivastava, and C. Cesarano (Aracne Editrice, Roma, 2001).

[4] L. Giannessi, Nuovo Cimento Soc. Ital. Fis. 112A, 447 (1999). See also L. Giannessi and M. Quattromini, “An overview of TREDI and CSR test cases, at www.desy.de/ csr/csr_workshop_2002/csr_workshop_2002_index.html

[5] R. Bonifacio, C. Pellegrini, and L. M. Narducci, Opt. Commun. 50, 373 (1984).

[6] J. D. Jackson, Classical Electrodynamics (John Wiley \& Sons, New York, 1975), p. 654.

[7] W. H. Press, B. P. Flannery, S. A. Teukolsky, and W.T. Vetterling, Numerical Recipes (Cambridge University Press, New York, 1988), p. 615.

[8] T. J. T. Kwan, IEEE J. Quantum Electron. 17, 1394 (1981).

[9] L. Serafini and J. B. Rosenzweig, Phys. Rev. E 55, 7565 (1997).

[10] B. E. Carlsten, Nucl. Instrum. Methods Phys. Res., Sect. A 285, 313 (1989).

[11] P. G. O'Shea, in Proceedings of the 2nd ICFA Advanced Accelerator Workshop, edited by J. B. Rosenzweig and L. Serafini (World Scientific, Singapore, 2000), p. 17.

[12] L. Serafini, in Proceedings of the 2nd ICFA Advanced Accelerator Workshop (Ref. [11]), p. 27.

[13] L. Serafini and C. Pagani, in Proceedings of the 1st EPAC Conference, Rome, 1988, edited by S. Tazzati (World Scientific, Singapore, 1988), p. 866.

[14] M. Borland, Summary of Equations and Methods Used in SPIFFE, APS/IN/LINAC/92-2, 1992, p. 29. The code SPIFFE is available on line at http://www.aps.anl.gov/asd/ oag/oagSoftware.shtml

[15] L. Giannessi and M. Quattromini, TREDI: A Self Consistent Three-Dimensional Integration Scheme for RF-Gun Dynamics Based on the Lienard-Wiechert Potentials Formalism, in Proceedings of the Workshop Towards X-Ray Free Electron Lasers, Gargnano, Italy, 1997, AIP Conf. Proc. No. 413 (AIP, New York, 1997), p. 313 (see also http://www.tredi.enea.it).

[16] J. L. Coacolo et al., Nucl. Instrum. Methods Phys. Res., Sect. A 393, 430 (1997).

[17] E. Colby, Ph.D. thesis, UCLA, 1997. LANL version: see, e.g., http://laacg1.lanl.gov/laacg/services/parmela.html, UCLA version: "Design, Construction and Testing of a Radiofrequency Electron Photoinjector for the Next Generation Linear Collider."

[18] M. J. de Loos and S. B. van der Geer, in Proceedings of the 5th European Particle Accelerator Conference, Sitges, 1996 (IOP, Bristol, 1996), p. 1241. 
[19] GPT User Manual, Pulsar Physics, Flamingostraat 24, 3582 SX Utrecht, The Netherlands (http://www. pulsar.nl).

[20] K. Floettmann, Astra User Manual, see http://www. desy.de/ mpyflo/Astra_dokumentation/

[21] K. J. Kim, Nucl. Instrum. Methods Phys. Res., Sect. A 275, 201 (1989).

[22] K. Floettmann (private communication).

[23] M. Ferrario et al., Part. Accel. 52, 1 (1996).

[24] M. Ferrario, J.E. Clendenin, D. T. Palmer, J. B. Rosenzweig, and L. Serafini, in Proceedings of the 2nd ICFA Advanced Accelerator Workshop (Ref. [11]), p. 534.

[25] I. M. Kapchinskj and V.V. Vladimirsky, in Proceedings of the II International Conference on High Energy Accelerators \& Instrumentation, Geneva, 1959 (CERN, Geneva, 1959), p. 274.

[26] M. Ferrario (private communication).

[27] L. Serafini (private communication).

[28] M. Ferrario, K. Floettmann, B. Grygorian, T. Limberg, and Ph. Piot, TESLA Report No. TESLA-FEL 2001-03, 2001.

[29] L. Giannessi and M. Quattromini, in Proceedings of the ICFA Workshop "The Physics and Applications of High Brightness Electron Beams," Chia Lagune, Sardinia, 2002, edited by L. Serafini, G. Travish, and J. Rosenzweig (World Scientific, Singapore, to be published).

[30] E. Colby, V. Ivanov, Z. Li, and C. Limborg, in Proceedings of the International Computational Accelerator Physics Conference, East Lansing, MI, 2002 (to be published).

[31] "User/Configurable MAGIC for electromagnetic PIC calculations," Comput. Phys. Commun. 87, 54-86 (1995). Information may be found at http://www.mrcwdc.com

[32] C. Limborg et al., in Proceedings of the 2003 Particle Accelerator Conference, Portland (IEEE, Piscataway, NJ, to be published).

[33] P. Piot, in Proceedings of the ICFA Workshop "The Physics and Applications of High Brightness Electron Beams," Chia Lagune, Sardinia, 2002 (Ref. [29]).

[34] J. Yang et al., in Proceedings of the VIII European Particle Accelerator Conference, Paris, 2002 (EPSIGA/CERN, Geneva, 2002).

[35] SLAC Report No. SLAC-R-593 UC-414, 2002.

[36] DESY Report No. TDR-2001-23, 2001.

[37] L. Serafini and M. Ferrario, AIP Conf. Proc. 581, 87 (2001).

[38] M. Ferrario et al., in Proceedings of the VIII European Particle Accelerator Conference, Paris, 2002 (Ref. [34]).

[39] E. L. Saldin, E. A. Schneidmiller, and M.V. Yurkov, Nucl. Instrum. Methods Phys. Res., Sect. A 398, 373 (1997).

[40] E. L. Saldin et al., Nucl. Instrum. Methods Phys. Res., Sect. A 483, 516 (2002).

[41] S. Heifets et al., SLAC Report No. SLAC-PUB-9165, 2002.

[42] Z. Huang, K. J. Kim, Phys. Rev. ST Accel. Beams 5, 074401 (2002).

[43] S. Reiche and. B. Rosenzweig, in Proceedings of the ICFA Workshop "The Physics and Applications of High
Brightness Electron Beams," Chia Lagune, Sardinia, 2002 (Ref. [29]).

[44] A. Kabel, M. Dohlus, and T. Limberg, Nucl. Instrum. Methods Phys. Res., Sect. A 455, 185 (2000).

[45] M. Dohlus, A. Kabel, and T. Limberg, Nucl. Instrum. Methods Phys. Res., Sect. A 445, 338 (2000).

[46] R. Li, Nucl. Instrum. Methods Phys. Res., Sect. A 429, 310 (1998).

[47] W. H. Press, B. P. Flannery, S. A. Teukolsky, and W.T. Vetterling, Numerical Recipes in C: The Art of Scientific Computing (Cambridge University Press, New York, 1992), 2nd ed., p. 309.

[48] A. Kabel, SLAC Report No. SLAC-PUB-9352, 2002.

[49] M. Borland, Phys. Rev. ST Accel. Beams 4, 070701 (2001).

[50] M. Borland, computer code ELEGANT, ANL, http:// www.aps.anl.gov/asd/oag/oaghome.shtml

[51] P. Emma (private communication).

[52] M. Dohlus (private communication).

[53] P. Emma, in Proceedings of the VIII European Particle Accelerator Conference, Paris, 2002 (Ref. [34]).

[54] P. Emma (private communication).

[55] Proceedings of the ICFA Beam Dynamics Mini Workshop on "Coherent Synchrotron Radiation and its Impact on the Dynamics of High Brightness Electron Beams," DESY-Zeuthen, 2002, www.desy.de/csr/ csr_workshop_2002/csr_workshop_2002_index.html

[56] L. Giannessi and M. Quattromini (to be published).

[57] L. R. Elias and I. Kimel, Nucl. Instrum. Methods Phys. Res., Sect. A 393, 100 (1997).

[58] L. Giannessi, P. Musumeci, and M. Quattromini, Nucl. Instrum. Methods Phys. Res., Sect. A 436, 443 (1999).

[59] W. B. Colson, Nucl. Instrum. Methods Phys. Res., Sect. A 393, 82 (1997).

[60] T. M. Tran and J. S. Wurtele, Comput. Phys. Commun. 54, 263 (1989).

[61] R. A. Jong, W. M. Fawley, and E.T. Scharlemann, in Modeling and Simulation of Laser Systems, SPIE Proc. Vol. 1045 (SPIE-International Society for Optical Engineering, Bellingham, WA, 1989), pp. 18-27.

[62] Z. Huang and W. M. Fawley, in Proceedings of the 2001 Particle Accelerator Conference, Chicago (IEEE, Piscataway, NJ, 2001), p. 2713.

[63] B. Faatz, W. M. Fawley, P. Pierini, S. Reiche, G. Travish, D. Whittum, and J. Wurtele, Nucl. Instrum. Methods Phys. Res., Sect. A 393, 277 (1997).

[64] B. D. McVey, Nucl. Instrum. Methods Phys. Res., Sect. A 250, 449 (1985).

[65] C. G. Parazzoli, in Proceedings of the X-Ray FEL Theory and Simulation Codes Workshop, Stanford Linear Accelerator Center, 1999 (SLAC Report No. LCLS-TN-00-1, 1999), p. 161.

[66] S. Reiche, Nucl. Instrum. Methods Phys. Res., Sect. A 429, 243 (1999).

[67] H. P. Freund, S. G. Biedron, and S.V. Milton, IEEE J. Quantum Electron. 36, 275 (2000).

[68] S. G. Biedron, H. P. Freund, and S.V. Milton, in FreeElectron Laser Challenges II, edited by Harold E. Bennett and David H. Dowell, SPIE Proc. Vol. 3614 (SPIE-International Society for Optical Engineering, Bellingham, WA, 1999). 
[69] P. Sprangle, A. Ting, and C. M. Tang, Phys. Rev. A 36, 2773 (1987).

[70] P. Sprangle, A. Ting, and C. M. Tang, Phys. Rev. Lett. 59, 202 (1987).

[71] G. Dattoli, L. Giannessi, P. L. Ottaviani, S. G. Biedron, H. P. Freund, and S.V. Milton, Opt. Commun. (to be published)

[72] G. Dattoli, M. Galli, and P. L. Ottaviani, ENEA Report No. RT/INN/93/09, 1993, p. 5.

[73] N. M. Kroll, L. P. Morton, and M. N. Rosenbluth, IEEE J. Quantum Electron. 17, 1436 (1981).

[74] W. B. Colson, in Classical Free Electron Laser Theory, edited by W. B. Colson, C. Pellegrini, and A. Renieri, Laser Handbook Vol. VI (North-Holland, Amsterdam, 1985).

[75] An example of numerical integration of the FEL pendulum equation is given in Perseo, http://www.perseo. enea.it

[76] G. Dattoli, L. Giannessi, and A. Torre, J. Opt. Soc. Am. B 10, 2136 (1993).

[77] C. Penman and B.W. J. McNeil, Opt. Commun. 90, 82 (1992).

[78] W. M. Fawley, Phys. Rev. ST Accel. Beams 5, 070701 (2002).

[79] G. Dattoli, A. Renieri, A. Torre, and R. Caloi, Nuovo Cimento Soc. Ital. Fis. 11D, 393 (1989).

[80] M. Xie, in Proceedings of the Particle Accelerator Conference, Dallas, TX, 1995 (IEEE, Piscataway, NJ, 1996), p. 183.

[81] G. Dattoli, L. Giannessi, P. L. Ottaviani, and M. Carpanese, Nucl. Instrum. Methods Phys. Res., Sect. A 393, 133 (1997).

[82] G. Dattoli and P. L. Ottaviani, Opt. Commun. 204, 283 (2002).

[83] J.W. Lewellen et al., Nucl. Instrum. Methods Phys. Res., Sect. A 483, 40 (2002).

[84] S. G. Biedron et al., Nucl. Instrum. Methods Phys. Res., Sect. A 445, 110 (2000).

[85] A. Tremaine et al., Nucl. Instrum. Methods Phys. Res., Sect. A 483, 24 (2002).
[86] S. Reiche, in Proceedings of the ICFA Workshop "The Physics and Applications of High Brightness Electron Beams," Chia Lagune, Sardinia, 2002 (Ref. [29]).

[87] R. Bonifacio et al., Nucl. Instrum. Methods Phys. Res., Sect. A 293, 627 (1990).

[88] R. Barbini et al., in Proceedings of Prospects for a $1 \AA$ FEL Laser, Sag Harbor, New York, 1990, edited by J. Gallardo (BNL Report No. 52273, 1990).

[89] R. Bonifacio et al., Nucl. Instrum. Methods Phys. Res., Sect. A 296, 787 (1990).

[90] L. H. Yu, Phys. Rev. A 44, 5178 (1991).

[91] F. Ciocci et al., IEEE J. Quantum Electron 31, 1242 (1995).

[92] L. H. Yu et al., Science 289, 932 (2000); S. G. Biedron, H. P. Freund, Z. Huang, K.-J. Kim, and S.V. Milton, in Proceedings of the 2001 Particle Accelerator Conference, Chicago (Ref. [62]), pp. 2704-2706.

[93] J. H. Wu and L. H. Yu, Nucl. Instrum. Methods Phys. Res., Sect. A 475, 104 (2001).

[94] W. Brefeld et al., Nucl. Instrum. Methods Phys. Res., Sect. A 483, 80 (2002).

[95] H. P. Freund and P. G. O'Shea, Nucl. Instrum. Methods Phys. Res., Sect. A 483, 449 (2002).

[96] H. P. Freund, S. G. Biedron, and S.V. Milton, Nucl. Instrum. Methods Phys. Res., Sect. A 445, 53 (2000).

[97] S. G. Biedron et al., Nucl. Instrum. Methods Phys. Res., Sect. A 483, 101 (2002).

[98] S. G. Biedron et al., Phys. Rev. ST Accel. Beams 5, 030701 (2002).

[99] Definitions and libraries for SDDS implementation may be found at the link http://www.aps.anl.gov/asd/oag/ oagPackages.shtml

[100] Definitions and libraries for HDF5 implementation may be found at the link http://hdf.ncsa.uiuc.edu/

[101] M. Borland et al., Nucl. Instrum. Methods Phys. Res., Sect. A 483, 268 (2002).

[102] S. Reiche, C. Pellegrini, J. Rosenzweig, P. Emma, and P. Krejcik, Nucl. Instrum. Methods Phys. Res., Sect. A 483, 70 (2002). 